\title{
HIV as an index stressor for PTSD: challenges and pitfalls in applying DSM criteria
}

HIV/AIDS carries a heavy burden for society, with between 31.4 and 35.3 million people infected and/or living with HIV/AIDS globally in 2009 and the largest proportion of these individuals living in Sub-Saharan Africa (22.5 million). ${ }^{1}$ At an individual level, the disease is associated with significant physical and psychiatric sequelae. The latter notably includes commonly occurring disorders of major depression and posttraumatic stress disorder (PTSD), with consistently high rates of PTSD (in the order of 13\% to 64\%) documented in HIV samples and data drawn from several studies supporting the validity of an HIV diagnosis as a PTSD qualifying event. ${ }^{2-5}$

\section{The trauma-PTSD relationship}

Since PTSD was first introduced as a disorder in 1980, several iterations to the diagnostic criteria have occurred. ${ }^{4}$ The current edition of the Diagnostic and Statistical Manual (DSM-IV) (APA) requires, most importantly, that a traumatic event of sufficient magnitude be experienced (Criterion Al). This experience must then be accompanied by a specified reaction (affect) (Criterion A2) and result in one or more symptoms of reexperience, three or more symptoms of avoidance, and two or more symptoms of hyperarousal (Criterion B-D respectively). If these symptoms cause the individual clinically significant distress (Criterion F) and have lasted at least one month (Criterion E) a diagnosis for PTSD can be made. ${ }^{6}$ Even though the DSM-IV-TR does not attempt to give an aetiological description of PTSD, it does presume that a traumatic event underlies the aetiology of the disorder. ${ }^{6}$ This makes the DSMIV-TR diagnostic structure for PTSD comparable to a conditioning 'stimulus-response' model, with the diagnostic manual assuming that a particular past, albeit recent past, traumatic event has occurred and that this event is responsible for the development of the disorder. ${ }^{6}$

With the release of the fourth edition of the DSM in 1994, life threatening disease was included as a qualifying trauma. ${ }^{6}$ In contrast, the DSM-III-R did not regard HIV as a PTSD qualifying event, as life threatening disease was not considered an event outside of the range of normal human experience which was then thought fundamental to the aetiology of PTSD 4 Of the medical illnesses that have since been studied in the realm of trauma and PTSD, some of the highest PTSD rates have been found in patients treated in intensive care units (ICUs) and in those with HIV infection. ${ }^{5}$

\section{Trauma and PTSD in HIV}

Owing in large part to the unique aetiological nature of PTSD and inclusion of life threatening disease to the criteria, profound challenges of a diagnostic and methodological nature have arisen. ${ }^{7}$ This has given rise to the question of whether HIV/AIDS is eligible as a qualifying event for PTSD. The psychological and psychiatric impact of HIV on infected individuals and comparison of prevalence studies assessing this impact has proven to be problematic. For example, medical and antiretroviral treatment advances have been substantial throughout the years and for infected individuals this means that 'being diagnosed with HIV' and/or 'living with HIV/AIDS' had a different impact in the past than it does now. The disease has changed from a death sentence to living with a chronic disease, which is especially true in developed countries where HIV treatments are more readily available. ${ }^{8}$

Another difficulty which confounds comparison across studies is that, although higher rates have been documented in HIV samples compared to healthy samples ${ }^{2}$, many of these studies have failed to make the clear and important distinction between PTSD secondary to general traumatic stressors (i.e. non-HIV stressors) and PTSD secondary to those that are HIV specific. ${ }^{2}$ In addition, a number of studies have based a diagnosis of PTSD on the presumed traumatic effect of receipt of an HIV positive diagnosis. ${ }^{3,9}$ While receipt of HIV positive status does comply with the stressor criterion of being diagnosed with a life threatening disease $(\mathrm{Al})$ and can lead to intense fear, helplessness, and horror (A2) especially if this news is unexpected ${ }^{8}$, it is unlikely that core PTSD symptom criteria (B-D) can be anchored to the notification of HIV positive status alone. ${ }^{\text {? }}$

Technically, to make a diagnosis of PTSD on the basis of receipt of HIV positive status, criteria A and B-D should be assessed on the basis of different but related stressors. Theoretically this means that two different criteria sets should be used to arrive at an HIV related PTSD diagnosis; the qualifying event ( $\mathrm{Al}$ and $\mathrm{A} 2$ ) and the consequences of HIV that lead to symptoms (B-D). This brings into question the issue of whether receipt of an HIV positive diagnosis alone can of itself cause or contribute to PTSD. HIV positive individuals are often exposed to the consequences of HIV such as access to treatment, the welfare of dependants, stigma, discrimination, physical decline and death ${ }^{3,4,9}$ and it is these consequences of an HIV positive status (including the loss of health and worries about children and family affected by HIV related mortality/morbidity), not the notification of status alone, that may eventually lead to the development of psychopathology.? The aforementioned stressors concern events that have yet to take place and are not anchored to one aetiological event in the past. Thus, individuals with HIV-specific traumatic experiences are unlikely to have recurrent and intrusive distressing recollections or experience symptoms of 
avoidance and hyper arousal arising from the diagnosis of HIV specifically, (criteria B-D). Instead these symptom criteria (BD) are most likely to be a result of the unfolding of future events related to their HIV positive status. ${ }^{\text {? }}$

\section{Traumatic Events in HIV}

In addition, a multitude of variables, that become 'stacked' over time, may together be significantly stressful for HIV infected individuals and cause posttraumatic symptomatology. These include treatment side effects from antibiotics and antiretrovirals, severity of psychiatric co-morbidities (e.g. adjustment disorder and depression), medication adherence as an impact to perceived life-style changes and to change in quality of life, CD4 cell counts and HIV viral loads, witnessing HIV related death, receipt of medical treatment, self-disclosure of HIV positive status, discrimination, and community ostracization. ${ }^{2,3,7,9,10}$

Thus, we argue that (i) living with HIV consists of a multitude of stressors rather than a single event; (ii) it is the consequence of a positive diagnosis (such as the loss of health and worries about children and family affected by HIV related mortality/morbidity) and not the notification of status alone, that may eventually lead to the development of clinically significant psychopathology and PTSD specifically; and (iii) HIV/AIDS is not a traumatic event that limits itself to one point in the past but represents an on-going traumatic experience. The lag in onset of PTSD symptoms found in HIV patients supports this hypothesis ${ }^{3}$, although it should be noted that longitudinal studies have been few and are in need of replication. Further, the link between the point of diagnosis and PTSD onset has been poorly substantiated. . $^{3,4} 8$ That said, the relatively high rates of PTSD in HIV-infected populations cannot simply be discounted or attributed to misdiagnosis or to symptoms characterising other disorders.

Another point of critique towards the eligibility of HIV as a qualifying event for PTSD is that the intense emotional reaction and horror should be considered as a normal human reaction. However, as Brewin, Lanius, Novac, Schnijder, Galea ${ }^{11}$ have pointed out, how much trauma-related functional impairment and symptom distress is 'normal' before it crosses the line towards pathology? This is underscored by findings indicating that even subthreshold PTSD syndromes are sufficient to cause significant functional impairment. ${ }^{12}$ We are thus faced with the dilemma of pathologising patients who may be expressing healthy reactions to a traumatic event, or alternatively failing to acknowledge pathological traumatisation due to HIV. The latter potentially leaves patients undiagnosed, untreated and without support and this is a risk that should not be underestimated considering that there is good evidence of PTSD leading to poorer medication adherence, more risk taking behaviour, and faster disease progression. ${ }^{10}$

In summary, there is little doubt that an HIV positive diagnosis can lead to psychological distress in the immediate aftermath of diagnosis and its associated consequences, while the related biological, psychological, and social sequelae of HIV may influence already present predispositions for possible psychopathology in some patients. Studies concerning the validity of HIV related PTSD are few and, in discussing prevalence rates of HIV-related PTSD, differences in sample characteristics, methodological assessments and instrumentation make it difficult to directly compare across studies. In addition, there remains a need for more efficient and psychometrically sound measuring instruments with high sensitivity and specificity to delineate PTSD syndromes in HIV, specifically with respect to onset and temporal course. PTSD assessment tools that are available are generic measures that have not been tailored to HIV samples. Although, we are not questioning the possible traumatic effects of living with HIV/AIDS and the possible emotional and psychological strains the disease brings with it, nor challenging the validity of currently available data on the topic, we are questioning the eligibility of HIV/AIDS as a qualifying event for a PTSD diagnosis as prescribed by the current DSM. ${ }^{6}$ Future studies investigating biological, social, cultural and psychological factors associated with trauma and PTSD in HIV samples may illuminate the nature of these various influences as well as provide more robust data on the differential weighting of the multitude of traumatic events in terms of their contribution to PTSD rates in HIV.

\section{Susanne Yvette Bakelaar', David Rosenstein', Ashraf Kagee ${ }^{2}$, Soraya Seedat ${ }^{1}$}

1Department of Psychiatry, Faculty of Health Sciences, Tygerberg Campus, Stellenbosch University, Stellenbosch, South Africa 2Department of Psychology, Stellenbosch University,

Stellenbosch, South Africa email: 16073371@sun.ac.za

\section{References}

1. UNAIDS. Available at: http://www.avert.org/worldstats.htm. Accessed 07/21, 2011.

2. Beckerman NL, Auerbach C. Post-traumatic stress disorder and HIV: a snapshot of co-occurrence. Soc Work Health Care 2010;49(8):687-702

3. Olley BO, Gxamza F, Seedat S, Theron H, Taljaard J, Reid E, et al. Psychopathology and coping in recently diagnosed HIVIAIDS patients-the role of gender. 2003.

4. Jones E, Wessely S. A paradigm shift in the conceptualization of psychological trauma in the 20th century. J Anxiety Disord 2007;21 (2):164-175.

5. Tedstone JE, Tarrier N. Posttraumatic stress disorder following medical illness and treatment. Clin Psychol Rev 2003;23(3):409-448.

6. American Psychiatric Association, American Psychiatric Association. Task Force on DSM-IV. Diagnostic and statistical manual of mental disorders: DSM-IV-TR. : American Psychiatric Publishing, Inc.; 2000.

7. Lucas GM. Antiretroviral adherence, drug resistance, viral fitness and HIV disease progression: a tangled web is woven. J Antimicrob Chemother 2005; 55(4):413

8. Safren SA, Gershuny BS, Hendriksen E. Symptoms of posttraumatic stress and death anxiety in persons with HIV and medication adherence difficulties. AIDS Patient Care STDS 2003;17(12):657-664.

9. Kagee A. Application of the DSM-IV Criteria to the Experience of Living with AIDS. Journal of Health Psychology 2008;13(8):1008.

10. Leserman J. Role of depression, stress, and trauma in HIV disease progression. Psychosom Med 2008;70(5):539.

11. Brewin CR, Lanius RA, Novac A, Schnyder U, Galea S. Reformulating PTSD for DSM V: Life after Criterion A. J Trauma Stress 2009;22(5):366-373

12. Van Hooff M, McFarlane AC, Baur J, Abraham M, Barnes DJ. The stressor Criterion-Al and PTSD: A matter of opinion? J Anxiety Disord 2009;23(1):77-86. 